\title{
Recent Standard Model measurements
}

\author{
Lucia Di Ciaccio*广 \\ Author affiliation: LAPP, Université Savoie Mont Blanc, CNRS/IN2P3, Annecy; France. \\ E-mail: Lucia.Di.Ciacciodcern.ch
}

\begin{abstract}
A selection of recent Standard Model measurements from ATLAS, CMS, D0 and HERA Collaborations is presented. The topics include studies of QCD with jets, photons and vector bosons as well as the latest measurements of the strong coupling constant. The status and prospects of precision measurements of the $W$-boson mass, the mixing angle of the electroweak interaction and the top-quark mass are also presented followed by recent results in the top-quark sector, on multibosons produced inclusively and on the study of the vector boson fusion and scattering. These measurements and studies provide stringent tests of the Standard Model. Deviations with respect to the Standard Model predictions are valuable probes of potential New Physics effects.
\end{abstract}

European Physical Society Conference on High Energy Physics - EPS-HEP2019 -

10-17 July, 2019

Ghent, Belgium

\footnotetext{
* Speaker.

${ }^{\dagger}$ Presenting results from ATLAS, CMS, LHCb, D0 and HERA Collaborations.
} 


\section{Introduction}

In the quest for a complete theory that describes at a fundamental level the observed phenomena, it is mandatory to perform measurements in order to understand until which point the Standard Model (SM) describes our world. At the same time, performing these measurements with high precision opens the possibility to find deviations with respect to the SM predictions accessing in this way an energy scale which may be beyond the reach of the present accelerators.

This paper reports about the SM results from ATLAS [1], CMS [2], LHCb [3], D0 [4] and HERA [5, 6] Collaborations, which are available less than a year after the end of the LHC Run 2. During this data-taking period a dataset of $140 \mathrm{fb}^{-1}$ was collected by ATLAS and CMS at an energy in the centre of mass $(\sqrt{s})$ of $13 \mathrm{TeV}$. Many of the presented measurements include already the full Run 2 statistics.

The measurements reported here would not have been possible without careful studies of the detector response and reconstruction performance leading to the use of improved algorithms and methods. They also profit from several steps forward in physics calculations and modelling.

\section{Test of QCD using jets, photons and vector bosons}

Cross section measurements of the jet production performed inclusively or in association with vector bosons are valuable tests of the QCD predictions and have the potential to constrain the Parton Distribution Functions (PDF). Two recent examples, which use the $\sqrt{s}=8 \mathrm{TeV}$ protonproton $(p p)$ dataset at the LHC, are the ATLAS measurement of the double-differential crosssection of the jet production in association with a $Z$ boson decaying into an electron-positron pair as a function of the jet rapidity and transverse momentum [7], and the CMS measurement of the associated production of a $W$ boson and a charm quark [8]. Figures 1a and $1 \mathrm{~b}$ illustrate some of the main results.

Figure 1a shows in particular that the Next-to-Next-to-Leading Order (NNLO) parton-level fixedorder predictions (corrected for hadronisation, underlying-event and QED radiation effects) improve the agreement with data and have a reduced uncertainty of about a factor two with respect to the Next-to-Leading Order (NLO) predictions. Fig. 1b shows that the predicted $\sigma\left(W^{+}+\right.$ $\bar{c}) / \sigma\left(W^{-}+c\right)$ ratio distribution depends on the choice of the PDF set and therefore the measurements provide a valuable input to constrain them.

Measurements of the jet production are also used to extract the strong coupling constant, $\alpha_{s}$, a crucial ingredient of perturbative QCD (pQCD) and the least-well known of the fundamental couplings [9]. The ATLAS Collaboration has performed measurements of $\alpha_{s}$ using the variable $R_{\Delta \Phi}$, which specifies the fraction of inclusive dijet events in which the azimuthal opening angle of the two jets, $\Delta \Phi$, is less than a specified value, $\Delta \Phi_{\max }[10]$. In the ratio the PDF dependence cancel to a large extent, and this allows an unbiased study of the $\alpha_{s}$ running. In this way the $\alpha_{s}$ evolution was measured up to an energy scale of $\sim 1.7 \mathrm{GeV}$ and found in agreement with the prediction from the renormalisation group equations. Recent $\alpha_{s}$ measurements are limited by the theoretical uncertainties related to the scale dependence of the fixed-order pQCD calculations. A recent determination of $\alpha_{s}$ at the scale of the Z-boson mass, $\alpha_{s}\left(M_{Z}^{2}\right)$, is obtained by the HERA Collaboration with a global NNLO QCD fit, performing a simultaneous extraction of the PDF and $\alpha_{s}$ using inclusive 


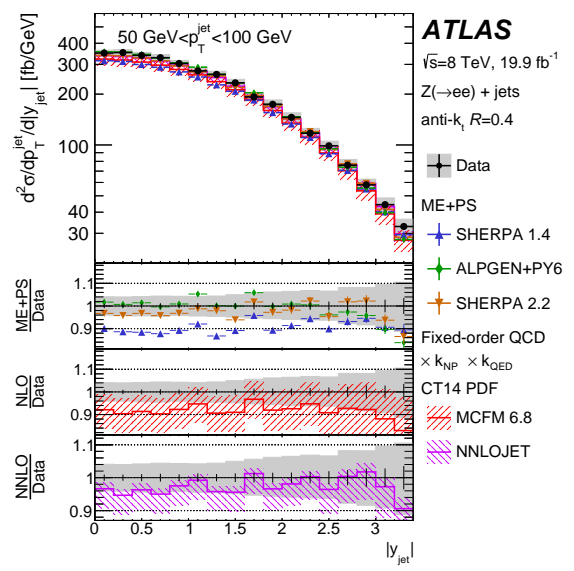

(a)

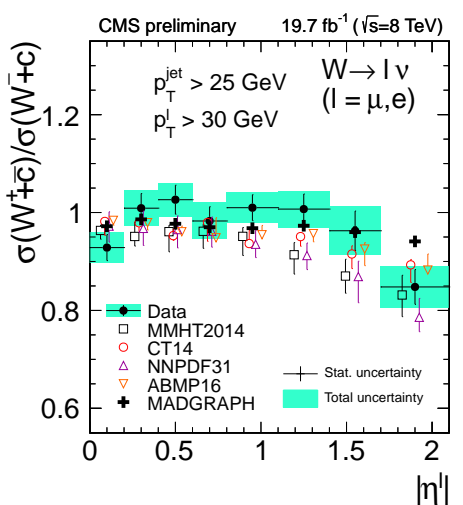

(b)

Figure 1: (a) The $Z+$ jets production cross-section as a function of the jet rapidity $\left(\left|y_{\text {jet }}\right|\right)$ in the jet transverse momentum range, $p_{\mathrm{T}}^{\mathrm{jet}}=50-100 \mathrm{GeV}$ [7]. The data are compared with parton shower $\mathrm{MC}$ generators and fixed-order theory predictions. (b) Cross section ratio $\sigma\left(W^{+}+\bar{c}\right) / \sigma\left(W^{-}+\right.$ $c$ ) as a function of the lepton pseudorapidity $\left(\eta^{1}\right)$ compared with theoretical predictions at NLO computed with MCFM and different PDF sets [8].

deep inelastic scattering (DIS) and jet data [11]. A 30\% reduction of the $\alpha_{s}\left(M_{Z}^{2}\right)$ scale uncertainty with respect to the NLO analyses is achieved. Recently the CMS Collaboration has measured $\alpha_{s}\left(M_{Z}^{2}\right)$ with a fit to the triple differential $t \bar{t}$ cross section calculated at NLO including also HERA DIS data [12]. In the fit $\alpha_{s}\left(M_{Z}^{2}\right)$, the top pole mass, $m_{t}^{\text {pole }}$ and PDFs are extracted simultaneously. The measured value of $\alpha_{s}\left(M_{Z}^{2}\right)$ is compatible with the previously mentioned HERA result and has a competitive total uncertainty.

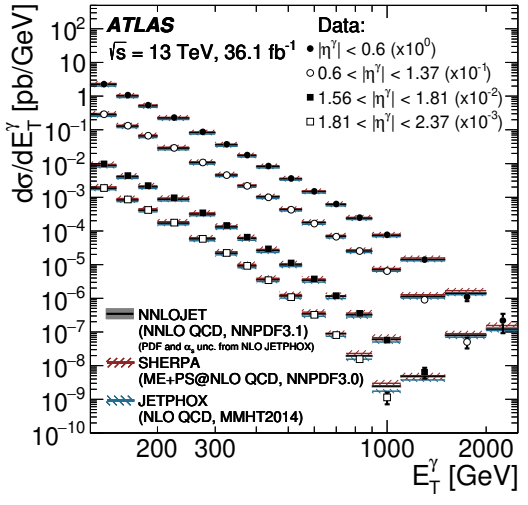

(a)



(b)

Figure 2: (a) The measured differential cross section for isolated-photon production as a function of the photon transverse energy, $\mathrm{E}_{\mathrm{T}}^{\gamma}$, in four photon-pseudorapidity $\left(\left|\eta^{\gamma}\right|\right)$ bins compared with theory predictions [13]. (b) The ratio of the NNLO and NLO QCD predictions to the measured differential cross section as a function of $\mathrm{E}_{\mathrm{T}}^{\gamma}$ for $\left|\eta^{\gamma}\right|<0.6$ [13]. 
The measurement of the production cross section of photons produced isolated or associated with jets tests $\mathrm{pQCD}$ and probes the gluon density in the proton. The recently published ATLAS analysis on isolated photons [13] performed at $\sqrt{s}=13 \mathrm{TeV}$ profits from improved photon calibration and identification procedures, which bring a reduced systematic uncertainty on the measured cross section of a factor up to $40 \%$. The cross section is measured as function of the photon transverse energy for different intervals of the photon pseudorapidity (Fig. 2a) and the comparison with predictions shows that recent NNLO calculations lead to a reduced theory scale uncertainty, (5\%) and to an improved description of the data, as it is illustrated in Fig. $2 b$.

The measurement of the transverse momentum of the electroweak (e.w.) gauge bosons, $p_{\mathrm{T}}^{V}$ ( $V=Z$, $W$ ), using the Drell-Yan process, tests several aspects of the strong interactions. At low $p_{\mathrm{T}}^{V}$, where fixed order calculations are unreliable due to soft and collinear parton radiation resulting in large logarithmic corrections, it tests resummed calculations. In the higher $p_{\mathrm{T}}^{V}$ regime it tests parton shower and fixed order predictions. A new $p_{\mathrm{T}}^{W}$ measurement [14] performed by the D0 Collaboration at $\sqrt{s}=1.96 \mathrm{TeV}$, where the $W$-boson production is dominated by valence quarks, focuses on the region below $p_{\mathrm{T}}^{W}=15 \mathrm{GeV}$, a region of special interest for e.w. precision measurements. The result is shown in Fig. 3a. A recent CMS analysis [15] measures the absolute and normalised $Z$ production cross section as function of $p_{\mathrm{T}}^{Z}$ from $300 \mathrm{MeV}$ up to few TeV using data collected at $\sqrt{s}$ $=13 \mathrm{TeV}$. Measurements of the cross section as function of $\Phi_{\eta}^{*}$, the angular correlations between Drell-Yan lepton pairs, stricly related to $p_{T}^{Z}$, are also provided.

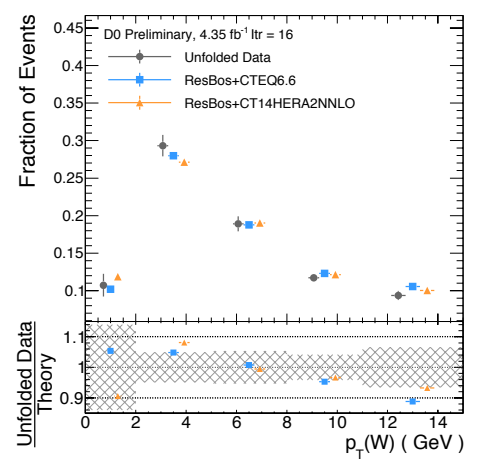

(a)

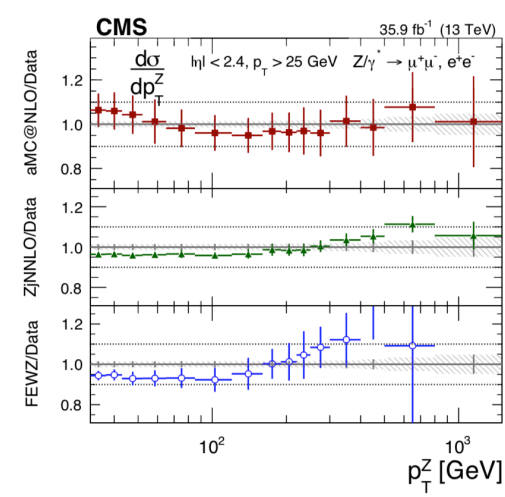

(b)

Figure 3: (a) The $W$ production cross-section as a function of the $W$ transverse momentum $\left(p_{\mathrm{T}}^{W}\right)$. The data are compared with RESBOS predictions and different PDF. The band in the ratio plot shown at the bottom, indicates the total experimental uncertainty [14]. (b) Ratio between the measured differential cross section for $Z$-boson production as function of its transverse momentum and different predictions. The total experimental uncertainty on the measurement is indicated by the hatched band [15] .

For $p_{\mathrm{T}}^{Z}<50 \mathrm{GeV}$ and $\Phi_{\eta}^{*}<0.5$ the experimental normalised cross section uncertainties are smaller than $0.5 \%$. The results indicate that predictions describe data within theory uncertainties: in particular at low $p_{\mathrm{T}}^{V}$ the RESBOS Monte Carlo featuring resummation of the logarithmically divergent terms at Next-to-Next-to-Leading Logarithmic (NNLL) accuracy, shows the best agreement with data but no theory uncertainties are available (Fig. 3a). At high $p_{\mathrm{T}}^{V}$ a good description is obtained 
with both MADGRAPH5 aMC@NLO and with Z+j-at-NNLO predictions (Fig. 3b). The latter includes the complete NNLO predictions of vector boson production in association with a jet and provides smaller uncertainties. A good understanding of the $p_{\mathrm{T}}^{V}$ distribution is a key ingredient for a precise measurement of the $W$-boson mass at the Tevatron and the LHC (see the referenced experimental publications for the citations of the MC and theory calculations).

\section{Electroweak precision measurements}

The mass of the $W$ boson, $m_{W}$, and the mixing angle of the e.w. interaction, $\theta_{W}$, are key parameters of the SM. They were very precisely measured at LEP, SLC, Tevatron, and more recently at the LHC (see [16] for a quick overview). In the e.w. theory, in the context of global fits to the SM parameters, they can be calculated from the $Z$-boson mass, $m_{Z}$, the fine-structure constant, $\alpha$, and the Fermi constant, $G_{\mu}$ when values of the top-quark and Higgs-boson masses are given. The comparison of the measured with the calculated values of $m_{W}$ and $\sin ^{2} \theta_{W}{ }^{1}$ tests the SM internal consistency and probes potential Beyond Standard Model (BSM) effects. Target uncertainties for precision future measurements are the SM prediction uncertainties from the e.w. fits: $\Delta m_{W} \leq 7 \mathrm{MeV}, \Delta \sin ^{2} \theta_{\text {eff }}^{\ell} \leq 6 \cdot 10^{-5}, \Delta m_{\text {top }} \leq 2 \mathrm{GeV}[16,17]$.

The total uncertainty in the combined LEP and Tevatron measurements of $m_{W}$ is $15 \mathrm{MeV}$ (see [19] for references). At LHC, ATLAS has measured $m_{W}$ with a total uncertainty of $19 \mathrm{MeV}$ using the $\sqrt{s}=7 \mathrm{TeV}$ dataset [19]. At hadron colliders the total uncertainty is dominated by the imprecise knowledge of the PDF and the modelling of QCD effects. To reduce the total uncertainty more refined experimental methods and improved theoretical predictions are necessary. Moreover a measurement of $m_{W}$ at forward rapidities (as proposed by the LHCb Collaboration) exploiting the partial anticorrelation of PDF with respect to existing measurements may help in reducing the uncertainty [20]. Ultimately, it is expected that with the Large Hadron Electron Collider the PDF uncertainty on $m_{W}$ will be reduced to $\approx 2 \mathrm{MeV}[18]$.

The $\sin ^{2} \theta_{\text {eff }}^{\ell}$ was measured very precisely at LEP and SLC but a tension exists between the two most precise measurements [16]. The uncertainty on the combined LEP and SLC measurement is: $\Delta \sin ^{2} \theta_{\mathrm{eff}}^{\ell}=1.6 \cdot 10^{-4}$ [21]. Although so far not as accurate as the lepton collider legacy result, the hadron-collider measurements with improved analyses may lead to competitive uncertainties. At hadron colliders two main methods are used: the first relys on fits to the forward-backward asymmetry in $Z\left(\rightarrow \ell^{-} \ell^{+}, \ell=e, \mu\right)$ decays, the second on the angular decomposition of the DrellYan cross-section in harmonic polynomials. At present the precision on $\sin ^{2} \theta_{\text {eff }}^{\ell}$ at hadron collider is limited by the imprecise knowledge of the PDF. A measurement of $\sin ^{2} \theta_{\mathrm{eff}}^{\ell}$ performed at high Zboson rapidity, like the one envisaged by the LHCb Collaboration [22], profits from the following two aspects: at high rapidity the forward-backward asymmetry is larger and the dilution effect related to the fact that at $p p$ colliders the quark direction is not known, is reduced. It is expected that at a future electron-positron collider, $\sin ^{2} \theta_{\text {eff }}^{\ell}$ will be measured with an uncertainty of $\Delta \sin ^{2} \theta_{\text {eff }}^{\ell} \sim$ $5 \cdot 10^{-6}[23]$.

\footnotetext{
${ }^{1}$ The analyses extract the effective leptonic weak mixing angle $\sin ^{2} \theta_{\mathrm{eff}}^{\ell}$ at the $m_{Z}$ scale where $\sin ^{2} \theta_{\mathrm{eff}}^{\ell}=\mathrm{k}_{f} \sin ^{2} \theta_{W}$ with $\mathrm{K}_{f}$ is a fermion dependent factor absorbing e.w. radiative corrections and $\sin ^{2} \theta_{W}$ is defined at tree level as 1 $m_{W}^{2} / m_{Z}^{2}$.
} 
As introduced above, the top-quark mass, $m_{t}$, is an important input for consistency tests of the SM. Precise measurement of $m_{t}$ are used to test the asymptotic behaviour of the vacuum potential. At present the most precise results rely on the reconstruction of the top-quark decay products. The method is defined as a 'direct' measurement. The ATLAS and CMS Collaborations achieve a total uncertainty below $0.5 \mathrm{GeV}$ combining their measurements of $m_{t}^{\text {direct }}$ [24]. Recent works estimate that the interpretation of $m_{t}^{\text {direct }}$ in terms of the pole of the top-quark propagator (pole mass, $m_{t}^{\text {pole }}$ ) is affected by a $0.5-1 \mathrm{GeV}$ uncertainty due to non-perturbative QCD effects [25, 26]. As an alternative approach, measurements using fits of the $t \bar{t}$ differential cross section and theory predictions were performed and provide the access to the top-quark mass $\left(m_{t}^{\text {pole }}\right)$ in a well defined mass scheme allowing a better understanding of the interpretation of the results obtained with both methods (see [27] for a compilation of recent $m_{t}^{\text {pole }}$ results). At present the most precise measurement of $m_{t}^{\text {pole }}\left(\Delta m_{t}^{\text {pole }}=0.8 \mathrm{GeV}\right)$ is obtained in a recent CMS analysis [12]; in a fit of the $t \bar{t}$ cross section, $m_{t}^{\text {pole }}$ is extracted simultaneously with the PDF and $\alpha_{s}$ using NLO predictions. From the point of view of the uncertainties the measurement of $m_{t}^{\text {direct }}$ and $m_{t}^{\text {pole }}$ are complementary: in the former, in most of the cases, the dominant uncertainty derives from the $b$-jet energy scale, in the latter the dominant uncertainties derives from the theory. Recently a new $m_{t}$ measurement has been performed by the CMS Collaboration using the $8 \mathrm{TeV}$ dataset [28]. It relies on a novel method to reconstruct highly-boosted top-quark decays. The top-quark mass is determined from the normalised distribution of the mass of a jet which includes all $t \rightarrow b W \rightarrow b q q^{\prime}$ products. Although so far not as accurate as the previous measurements, the Authors advocate that this method has a great potential and can be compared directly to precise analytical calculations, feasible only in the highly-boosted regime.

\section{Measurements with top quarks}

Precise measurements of top-quark production and decay properties provide crucial information for testing the expectations of the SM at a scale close to the Electroweak Symmetry Breaking (EWSB) scale and above. This section reports some of the very recent measurements in the top sector: the search for $t \bar{t} t \bar{t}$ events, the measurements of the top charge asymmetry and single-top results.

The production of four top quarks $(t \bar{t} t \bar{t})$ is a rare SM process but its cross section may be significantly enhanced in BSM theories [30, 31]. In SM, its study is a way to constrain the top Yukawa coupling. The latest results on the search for $t \bar{t} t \bar{t}$ events are published by the CMS Collaboration in the same charge dileptons and at least three lepton channels (electrons or muons) using the full $13 \mathrm{TeV}$ data sample [29]. It is a challenging search due to the small expected cross section $\left(\sigma_{S M}^{N L O}=12 \mathrm{fb}\right.$ in $p p$ collisions at $\left.\sqrt{s}=13 \mathrm{TeV}\right)$ and the many background processes, as illustrated in Fig. 4a. In spite of the use of the full Run 2 data sample, the process remains unobserved: the measured (expected) significance is 2.6 (2.7) standard deviations. The next step is to include the search results in additional final states and ultimately the Run 3 data. In this analysis of $t \bar{t} t \bar{t}$ events, the top Yukawa coupling is constrained to be $\left|y_{t}\right|<1.7$ at $95 \%$ confidence level with respect to its SM value. This result is comparable with recent CMS limits from $t \bar{t}$ kinematic distributions in lepton+jet and complementary to the coupling extraction in $t t H$ and $t H$.

According to the SM a small charge asymmetry is expected in $t \bar{t}$ events produced in $p p$ interactions. It results from the interference of higher order amplitudes of $q q$ and $q g$ initial states and manifests 
itself in the fact that the produced top quark prefers the initial-quark direction. The top charge asymmetry $\left(\mathrm{A}_{c}\right)$ is enhanced at high masses and longitudinal boost of the top-antitop pair. Some BSM theories predict an enhanced value of $\mathrm{A}_{c}$. The combined ATLAS and CMS measurement of the top charge asymmetry performed at $\sqrt{s}=8 \mathrm{TeV}$ is in agreement with NLO and NNLO SM predictions but also compatible with zero. The new ATLAS measurement of $\mathrm{A}_{c}$ using the full 13 $\mathrm{TeV}$ data sample [32], achieves for the first time an evidence of this effect (four standard deviations from zero) in agreement with NNLO QCD predictions with NLO EW corrections included. As expected in the $\mathrm{SM}, \mathrm{A}_{c}$ is enhanced at high $t \bar{t}$ masses as shown in Fig. $4 \mathrm{~b}$.



(a)

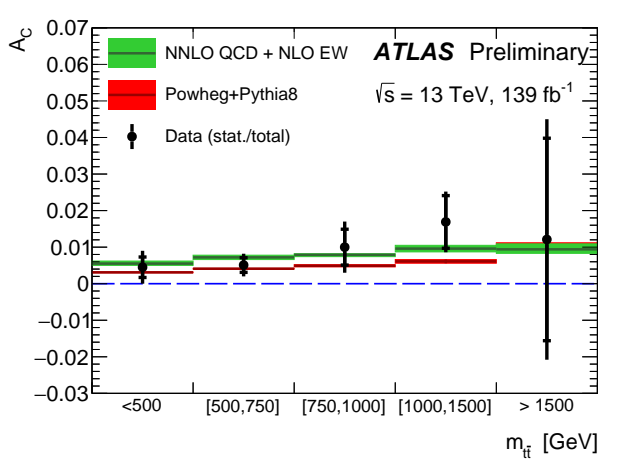

(b)

Figure 4: (a) Observed and expected multivariate discriminant distributions, BDT, after the statistical fit in the search for $t \bar{t} t \bar{t}$ events. The hatched areas represent the total post-fit uncertainties in the signal and background predictions. The lower panel shows the ratios of the observed event yield to the total prediction of signal plus background [29]. (b) Charge asymmetries in $t \bar{t}$ events as a function of the $t \bar{t}$ invariant mass, compared with SM theory predictions calculated at NNLO in QCD and NLO in EW theory (green) and with NLO parton shower MC generator predictions (red) [32].

The study of the single top-quark production plays an important role in indirect searches for new phenomena that could be modelled as anomalous couplings in an effective quantum field theory. In SM it provides a powerful probe for the e.w. coupling of the top quark. In the frame of the LHC top working group, the ATLAS and CMS Collaborations, have combined their single-top cross section measurements performed with $7 \mathrm{TeV}$ and $8 \mathrm{TeV}$ datasets [33] achieving an uncertainty of 7\% on the combined single top cross section measurement in the best measured channnel: the t-channel. This result is in agreement with the most precise SM prediction computed at NLO matched with NLL resummed calculations, which has a $3 \%$ uncertainty. The combined cross section results are then used to extract the value of the product of $V_{t b}$ times a form factor $f_{L V}$ accounting for possible BSM contributions to the $W t b$ vertex. The value of $\left|f_{L V} * V_{t b}\right|$ is compatible with 1 within a $4 \%$ total uncertainty. In spite of the small production cross section, thanks to the large data samples available at LHC, measurements of differential cross sections of the single-top production are possible. A recent CMS analysis [34] using the $13 \mathrm{TeV}$ dataset collected in 2015 and 2016 measures differential cross sections for t-channel single-top quark and antiquark production. The results demonstrate a good understanding of the underlying e.w. production mechanism of single-top quarks and in 
particular of the vector - axial-vector Lorentz structure of the coupling predicting highly polarized top quarks.

\section{Inclusive multiboson, vector boson fusion and scattering studies}

Measurements of the production of multibosons $(V V / V \gamma / \gamma \gamma$ or $V V V$ with $V=W, Z)$ investigate the non-abelian structure of the SM at high energy and are sensitive to new physics via anomalous Triple and Quartic Gauge Couplings (aTGC and aQGC). At the LHC several diboson production cross sections were measured inclusively in the number of jets at $\sqrt{s}=7,8$ and $13 \mathrm{GeV}$. The most recent CMS result is the measurement of the $Z Z$ cross section with the full $13 \mathrm{TeV}$ dataset [35]. A new ATLAS analysis studies the $Z \gamma$ production and measures several differential cross sections using the full $13 \mathrm{TeV}$ dataset [36] reaching a precisions of better than $5 \%$ in most measurement bins. Noteworthy is the measurement of the $W$ and $Z$ boson helicity fractions in $W Z$ events performed by the ATLAS Collaboration [37], which paves the way for future measurements of the scattering of longitudinally polarised gauge bosons. All results are consistent with SM predictions. The lesson learnt is that the NNLO theory predictions are necessary to have a good description of the experimental results and that the effect on the predictions of an additional order in the perturbative expansion (in this case the change between NLO and NNLO calculations) may be underestimated by the procedure used at LHC to evaluate the theory uncertainties relying on the variation of a factor one half and two of the QCD renormalisation scale.

In the triboson final state, recently, ATLAS has reported a $4.1 \sigma$ evidence in the search for $W V V, V=$ $W, Z$ combining the searches in the channels with two, three and four leptons. [38]. The CMS triboson search concentrates on the $W W W$ production using the same sign dilepton and trilepton channels and on the extraction of confidence intervals for aQGC [39].

Vector Boson Fusion (VBF) and Vector Boson Scattering (VBS) searches and measurements are milestone studies in the LHC physics program. They investigate the EWSB mechanism of the SM and are sensitive to new physics in the three or four boson vertices. The VBF and VBS processes are studied via the measurements of the e.w. production of a single gauge boson $(E W-V j j)$ or of a pair of gauge bosons $(E W-V V j j)$ and are characterised by a low jet activity in the central region and the presence of two jets with a large rapidity separation.

Processes mediated by the strong interactions resulting in a final state with two jets and one $(Q C D-$ $V j j)$ or two gauge bosons $(Q C D-V V j j)$ also exist and represent often the largest background. ATLAS and CMS have observed the $E W-V j j$ process with $V=W, Z$ and the $E W-V V j j$ process with $V V=W^{ \pm} W^{ \pm}$. ATLAS has also observed the $E W-W Z j j$ process with the $13 \mathrm{TeV}$ dataset collected in 2015 and 2016. Recently, a new ATLAS analysis using the full $13 \mathrm{TeV}$ dataset, reports the observation of the e.w. production of the $Z Z j j$ in the four lepton and two leptons plus two neutrino channels with a significance of $5.5 \sigma$ [40]. The result is obtained with a fit to a multivariable combination (BDT) as shown in Fig. 5. A new CMS study using the $13 \mathrm{TeV}$ dataset collected in 2015 and 2016 finds an evidence for the $E W-Z \gamma j j$ process [41]. The result is obtained with a fit to the invariant mass of the two leading jets in different intervals of the jet rapidity difference, as shown in Fig. 6. This result combined with the CMS result obtained with the $8 \mathrm{TeV}$ dataset achieves an observed significance of $4.7 \sigma$. 



Figure 5: Observed and expected multivariate discriminant distributions after the statistical fit in the $\ell \ell \ell \ell j j$ (left) and in the $\ell \ell v v j j$ (right) signal regions. The error bars on the data points show the data statistical uncertainty and the hashed bands represent the full uncertainties in the expectations [40].
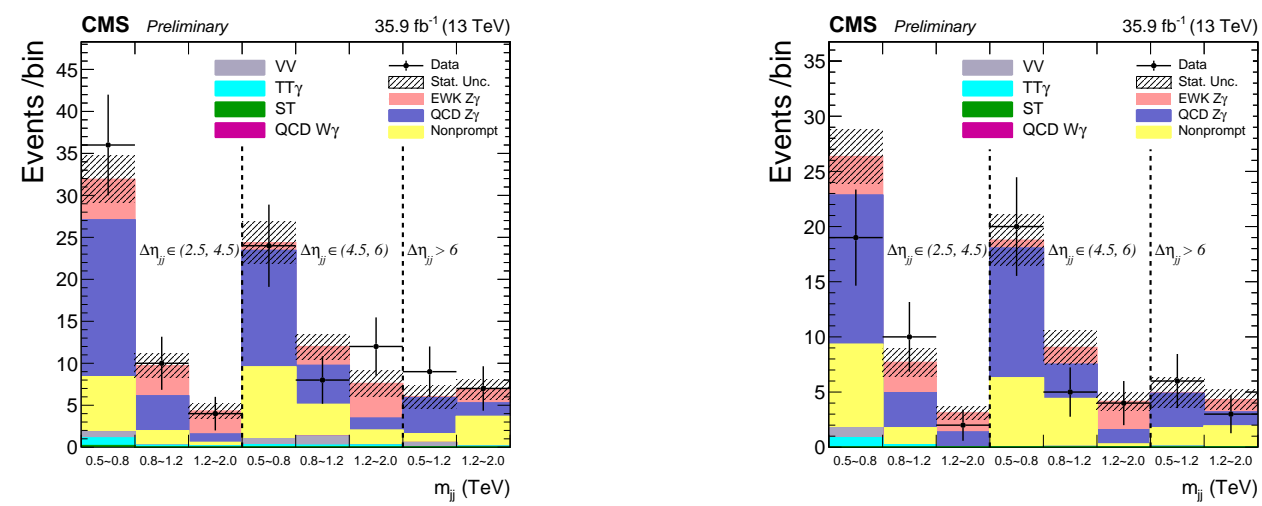

Figure 6: Observed and expected distributions of the di-jet invariant mass $\left(m_{j j}\right)$ in different $\Delta \eta_{j j}$ regions, after the statistical fit in the $\mu \mu \gamma j j$ (left) and the ee $\gamma j j$ (right) signal regions. The error bars on the data points show the data statistical uncertainty and the hashed bands represent the full uncertainties in the expectations [41].

Finally, in many of the multiboson and vector boson fusion and scattering studies, constraints on the structure of the triple and quartic vector boson interactions in the framework of a dimension- 6 and a dimension- 8 effective field theory (EFT) operators are extracted. The most stringent limits on the coefficients of the EFT operators are often obtained exploiting the hadronic decays of the gauge bosons [42].

\section{Conclusions}

Many recent SM results are available with increasing precision thanks to improved detector performance, experimental methods and to the large data sets collected at the LHC. Together with advances in theoretical calculations they provide stringent tests of the SM. 
Many integrated cross section results are limited by the systematic uncertainties, therefore improved analysis techniques and methods are required. Thanks to the large dataset available more and more differential cross section measurements are possible. For the interpretation of the results in the context of an EFT, the upper tail of the distributions retain the greatest sensitivity to BSM physics.

In order to increase the probability to find deviations with respect to the SM predictions caused by BSM effects, the interplay between e.w. precision, diboson, top and Higgs measurements must be exploited. Fits to the e.w. parameters and to the Wilson coefficients in the EFT approach may play an important role. The outcome of these studies will contribute to establish a solid basis for future projects in particle physics at colliders and beyond.

\section{References}

[1] ATLAS Collaboration, The ATLAS Experiment at the CERN Large Hadron Collider, JINST 3 (2008) S08003.

[2] CMS Collaboration, The CMS Experiment at the CERN LHC, JINST 3 (2008) S08004.

[3] LHCb Collaboration, The LHCb detector at the LHC, JINST 3 (2008) S08005.

[4] D0 Collaboration, The upgraded D0 detector, Nucl. Instrum. Methods A 565 (2006) 463, arXiv:physics/0507191[physics.ins-det].

[5] H1 Collaboration, The H1 detector at HERA, Nucl. Instrum. Meth. A386 (1997) 310.

[6] ZEUS Collaboration, The ZEUS Detector, Status Report, DESY (1993), doi:10.3204/PUBDB-2017-12635.

[7] ATLAS Collaboration, Measurement of the inclusive cross-section for the production of jets in association with a $\mathrm{Z}$ boson in proton-proton collisions at $8 \mathrm{TeV}$ using the ATLAS detector, arXiv:1907.06728[hep-ex].

[8] CMS Collaboration, Measurement of the associated production of a W boson and a charm quark at $\sqrt{s}=8 \mathrm{TeV}$, arXiv:CMS-PAS-SMP-18-013, https://cds.cern.ch/record/2682200.

[9] J. Kretzschmar, Standard Model physics at the LHC, Contribution to the volume "From my Vast Repertoire - the Legacy of Guido Altarelli", arXiv:1803.10800[hep-ex].

[10] ATLAS Collaboration, Measurement of dijet azimuthal decorrelations in pp collisions at $\sqrt{s}=8 \mathrm{TeV}$ with the ATLAS detector and determination of the strong coupling, Phys. Rev. D 98 (2018) 092004, arXiv:1805.04691 [hep-ex].

[11] H1, ZEUS and NNLOJET Collaborations HERAPDF2.0Jets NNLO (prel.), the completion of the HERAPDF2.0 family, H1prelim-19-041 ZEUS-prel-19-001, http://www-h1.desy.de/publications/htmlsplit/.

[12] CMS Collaboration, Measurement of $\mathrm{t} \overline{\mathrm{t}}$ normalised multi-differential cross sections in pp collisions at $\sqrt{s}=13 \mathrm{TeV}$, and simultaneous determination of the strong coupling strength, top quark pole mass, and parton distribution functions, arXiv:1904.05237 [hep-ex].

[13] ATLAS Collaboration, Measurement of the inclusive isolated-photon cross section in pp collisions at $\sqrt{s}=13 \mathrm{TeV}$ using $36 \mathrm{fb}^{-1}$ of ATLAS data, arXiv:1908.02746 [hep-ex]. 
[14] Measurement of the Shape of the Transverse Momentum Distribution of W Bosons produced in pp Collisions at $\sqrt{s}=1.96 \mathrm{TeV}$, D0 note 6508-CONF, https://www-d0.fnal.gov/Run2Physics/WWW/results/prelim/EW/E45/E45.pdf.

[15] CMS Collaboration, Measurements of differential Z boson production cross sections in pp collisions with CMS at $\sqrt{s}=13 \mathrm{TeV}$, arXiv:1909.04133 [hep-ex].

[16] J. Erler et al., Electroweak Precision Tests of the Standard Model after the Discovery of the Higgs Boson, Prog. Part. Nucl. Phys. 106 (2019) 68, arXiv:1902.05142 [hep-ex].

[17] J. Haller et al., Update of the global electroweak fit and constraints on two-Higgs-doublet models, Eur. Phys. J C78 (2018) 675, arXiv:1803.01853 [hep-ex].

[18] M. Klein, LHeC: Status and Prospects, these proceedings.

[19] ATLAS Collaboration, Measurement of the W-boson mass in pp collisions at $\sqrt{s}=7$ TeV with the ATLAS detector, Eur. Phys. J. C 78 (2018) 110, arXiv:1701.07240 [hep-ex].

[20] G Bozzi et al., Prospects for improving the LHC W boson mass measurement with forward muons, Eur. Phys. J. C 75 (2015) 60, arXiv:1508.06954[hep-ex].

[21] ATLAS Collaboration, Measurement of the effective leptonic weak mixing angle using electron and muon pairs from Z-boson decay in the ATLAS experiment at $\sqrt{s}=8 \mathrm{TeV}$, ATLAS-CONF-2018-037, http://cdsweb.cern.ch/record/2630340.

[22] LHCb Collaboration, Physics case for an LHCb Upgrade II - Opportunities in flavour physics, and beyond, in the HL-LHC era, arXiv:1808.08865[hep-ph].

[23] E Locci, Precision Electroweak Measurements at FCC-ee, These Proceedings.

[24] LHC top working group, Top Quark Mass, https://twiki.cern.ch/twiki/pub/LHCPhysics/LHCTopWGSummaryPlots/.

[25] P. Nason, The Top Mass in Hadronic Collisions, Contribution to the volume "From my Vast Repertoire - the Legacy of Guido Altarelli", arXiv:1712.02796[hep-ph].

[26] A. H. Hoang The Top Mass: Interpretation and Theoretical Uncertainties, Proceedings, 7th International Workshop on Top Quark Physics (TOP2014): Cannes, France, September 28-October 3, 2014, arXiv:1412.3649[hep-ph].

[27] ATLAS Collaboration, Measurement of the top-quark mass in t $\bar{t}+1$-jet events collected with the ATLAS detector in pp collisions at $\sqrt{s}=8 \mathrm{TeV}$, arXiv:1905.02302 [hep-ex].

[28] CMS Collaboration, Measurement of the jet mass distribution in highly boosted top quark decays in pp collisions at $\sqrt{s}=13 \mathrm{TeV}$ arXiv:CMS-PAS-TOP-19-005, https://cds.cern.ch/record/2682624.

[29] CMS Collaboration, Search for production of four top quarks in final states with same-sign or multiple leptons in proton-proton collisions at $\sqrt{s}=13 \mathrm{TeV}$, arXiv:1908.06463[hep-ex].

[30] D. Dicus et al., Higgs decay to top quarks at hadron colliders, Phys. Lett. B 333 (1994) 126, arXiv:hep-ph/9404359.

[31] N. Craig et al., Heavy Higgs bosons at low tan $\beta$ : from the LHC to $100 \mathrm{TeV}$, JHEP 01 (2017) 018, arXiv:1605.08744 [hep-ex].

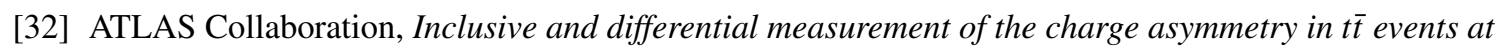
$13 \mathrm{TeV}$ with the ATLAS detector, ATLAS-CONF-2019-026, https://cds.cern.ch/record/2682109. 
[33] ATLAS and CMS Collaboration, Combinations of single-top-quark production cross-section measurements and $\left|f_{L V} * V_{t b}\right|$ determinations at $\sqrt{s}=7$ and 8 TeV with the ATLAS and CMS experiments, JHEP 05 (2019) 088, arXiv:1902.07158[hep-ph].

[34] CMS Collaboration, Measurement of differential cross sections and charge ratios for t-channel single top quark production in proton-proton collisions at $\sqrt{s}=13 \mathrm{TeV}$, arXiv:1907.08330.

[35] CMS Collaboration, Measurement of the $p p \rightarrow Z Z$ production cross section at $\sqrt{s}=13$ with the Run 2 data set, CMS-PAS-SMP-19-001, https://cds.cern.ch/record/2668717.

[36] ATLAS Collaboration, Measurement of $Z \gamma \rightarrow \ell^{+} \ell^{-} \gamma$ differential cross-sections in pp collisions at $\sqrt{s}$ $=13$ with the ATLAS detector, ATLAS-CONF-2019-034, https://cds.cern.ch/record/2682846.

[37] ATLAS Collaboration, Measurement of $W^{ \pm} Z$ production cross sections and gauge boson polarisation in pp collisions at $\sqrt{s}=13 \mathrm{TeV}$ with the ATLAS detector, Eur. Phys. J. C 79 (2019) 535, arXiv:1902.05759 [hep-ex].

[38] ATLAS Collaboration, Evidence for the production of three massive vector bosons with the ATLAS detector, arXiv:1903.10415.

[39] CMS Collaboration, Search for the production of $W^{ \pm} W^{ \pm} W^{\mp}$ at $\sqrt{s}=13$, Phys. Rev. D 100012004 , arXiv:1905.04246

[40] ATLAS Collaboration, Observation of electroweak production of two jets in association with a Z-boson pair, arXiv:ATLAS-CONF-2019-033, https://cds.cern.ch/record/2682845.

[41] CMS Collaboration, Measurement of electroweak production of $Z \gamma$ in association with two jets in proton-proton collisions at $\sqrt{s}=13 \mathrm{TeV}$, CMS-PAS-SMP-18-007, https://cds.cern.ch/record/2682214.

[42] Limits on anomalous triple and quartic gauge couplings, arXiv:http://cern.ch/go/8ghC 